\title{
SOME RECURSIVELY UNSOLVABLE PROBLEMS IN ANALYSIS
}

ANDREW ADLER

I. Systems of exponential polynomials. Tarski has observed in [2] that there is no decision procedure for the elementary theory of complex numbers with addition, multiplication, and exponentiation. In this section a stronger result is proved: there is no uniform procedure for deciding whether a system of equations built up using addition, multiplication, and exponentiation has a solution in complex numbers.

We write $\operatorname{Rat}(x)$ if $x$ is rational, $\operatorname{Int}(x)$ if $x$ is an integer, and $\operatorname{Nat}(x)$ if $x$ is a natural number. In what follows, unless otherwise specified, variables range over the complex numbers.

It is easy to verify that

$$
\operatorname{Nat}(x) \leftrightarrow \exp (2 \pi i x)=1 \wedge \exp (2 \pi i \exp (x \log 2))=1
$$

Martin Davis has shown in [1] that for every recursively enumerable predicate $Q(n)$, there exists a polynomial $P\left(n, x_{1}, \cdots, x_{k}, y\right)$ with integer coefficients such that

$$
Q(n) \leftrightarrow\left(\exists x_{1}, \cdots, x_{k}, y\right)\left(P\left(n, x_{1}, \cdots, x_{k}, y\right)=0 \wedge y=2^{x_{1}}\right)
$$

where now the variables range over the natural numbers.

Let $\Sigma_{n}$ be the system consisting of the following equations:

(i) $\exp \left(2 \pi i x_{j}\right)=1,1 \leqq j \leqq k$.

(ii) $\exp \left(2 \pi i \exp \left(x_{j} \log 2\right)\right)=1,1 \leqq j \leqq k$.

(iii) $y=\exp \left(x_{1} \log 2\right)$.

(iv) $P\left(n, x_{1}, \cdots, x_{k}, y\right)=0$.

It follows from (A) and (B) that $Q(n)$ holds iff the system $\Sigma_{n}$ has a solution in complex numbers. Let $Q(n)$ be in particular not recursive. Then there is no algorithm to determine, given $n$, whether the system $\Sigma_{n}$ has a solution in complex numbers.

The equations in the systems $\Sigma_{n}$ do not all have integer coefficients. This defect can be remedied. For

(a) $\operatorname{Rat}(x) \leftrightarrow(\exists u, v)(\exp (u)=1 \wedge \exp (x u)=1 \wedge u v=1)$.

Let the predicate $S(y, x)$ be defined by

(b) $S(y, x) \leftrightarrow(\exists w)(\exp (w)=2 \wedge y=\exp (x w))$.

Then we have

(c) $\operatorname{Int}(x) \leftrightarrow(\exists y)(S(y, x) \wedge \operatorname{Rat}(x) \wedge \operatorname{Rat}(y))$.

For if $x$ is rational, $S(y, x)$ iff $y=2^{x} \exp (2 \pi i n x)$ for some integer $n$. If

Received by the editors August 21, 1968. 
$y$ is rational, we must have $\exp (2 \pi i n x)= \pm 1$. When $x$ is rational, $\pm 2^{x}$ is rational iff $x$ is an integer. But

(d) $\operatorname{Nat}(x) \leftrightarrow(\exists y)(S(y, x) \wedge \operatorname{Int}(x) \wedge \operatorname{Int}(y))$ and

(e) If $x$ is a natural number, then $y=2^{x_{\leftrightarrow}} S(y, x) \wedge$ Nat $(y)$.

From (a), (b), (c), (d), (e) and (B), it is now easy to construct systems $\Sigma_{n}^{\prime}$ with integer coefficients such that $\Sigma_{n}^{\prime}$ has a solution in complex numbers iff $Q(n)$ holds. Hence we have proved

THEOREM I. There is no uniform procedure for deciding whether a system of equations with integer coefficients, built up by addition, multiplication, and exponentiation has a solution in complex numbers.

By way of contrast, it is not known if there is a procedure for deciding whether a system of equations of the kind described in Theorem I has a solution in real numbers. The situation changes if we allow complex coefficients, for the systems $\Sigma_{n}$ described in this section have a solution in complex numbers iff they have a solution in real numbers.

II. Systems of algebraic differential equations. In this section it is shown that there is no uniform decision procedure for whether a system of algebraic differential equations with integer coefficients has a solution in real-valued functions defined in the interval $[0,1]$. Lower case Latin letters will denote in this section functions defined in $[0,1]$ of the real variable $\alpha$.

It is easy then to verify that:

$$
\text { (C) } \begin{aligned}
\operatorname{Nat}(x) \leftrightarrow(\exists w, f)\left(x=w^{2} \wedge x^{\prime}=0\right. & \wedge f^{\prime \prime}+\pi^{2} x^{2} f=0 \wedge f(0) \\
& \left.=0 \wedge f^{\prime}(0)=1 \wedge f(1)=0\right) .
\end{aligned}
$$

If $x$ is a constant, then

(D) $y=2^{x} \leftrightarrow(\exists g)\left(g^{\prime}=(x \log 2) g \wedge g(0)=1 \wedge y^{\prime}=0 \wedge g(1)=y(1)\right)$.

Let $\Delta_{n}$ be the system consisting of the following equations with boundary conditions:

(i) $x_{i}=w_{i}^{2} ; \quad x_{i}^{\prime}=0 ; f_{i}^{\prime \prime}+\pi^{2} x_{i}^{2} f_{i}=0 ; \quad f_{i}(0)=0 ; f_{i}^{\prime}(0)=1 ; f_{i}(1)=0$ $(i=1, \cdots, k)$.

(ii) $g^{\prime}=\left(x_{1} \log 2\right) g ; g(0)=1 ; y^{\prime}=0 ; g(1)=y(1)$.

(iii) $P\left(n, x_{1}, \cdots, x_{k}, y\right)=0$.

Then from (B), (C), (D), the predicate $Q(n)$ of $\$$ I holds iff the system $\Delta_{n}$ has a solution in real-valued functions defined in $[0,1]$.

By altering our systems slightly, we can ensure that all the coefficients are integers. For $s=\log 2$ iff $s^{\prime}=0$ and there exists a function $h$ such that $h^{\prime}=s h, h(0)=1, h(1)=2$. Also $t=\pi$ iff $t^{\prime}=0$ and there is 
a function $k$ such that $k^{\prime \prime}+t^{2} k=0, k(0)=0, k^{\prime}(0)=1$, and $k(1)=0$, where further $0 \leqq t \leqq 4$. But $0 \leqq t \leqq 4$ iff $(\exists p, q)\left(t=p^{2} \wedge p^{2}+q^{2}=4\right)$. All the boundary conditions can also be removed, at the expense of introducing new function variables. For in general if $m$ and $n$ are constants, then $f(m)=n$ iff $(\exists g)(f-n=(m-\alpha) g)$. Hence we can in a simple way construct systems $\Delta_{n}^{\prime}$ of algebraic differential equations with integer coefficients and no boundary conditions such that $\Delta_{n}^{\prime}$ has a solution in real-valued functions defined on $[0,1]$ iff $Q(n)$ holds. Proceeding as in $\S \mathrm{I}$, we have proved

THEOREM II. There is no uniform procedure for deciding whether a system of algebraic differential equations with integer coefficients has a solution in real-valued functions defined on $[0,1]$.

In the construction of the systems $\Delta_{n}$, equation (iii) can be replaced by

(iii) ${ }^{*} P\left(n, x_{1}(1), \cdots, x_{k}(1), g(1)\right)=0$.

The equations involving $y$ can then be removed from (ii). All the other boundary conditions of the systems $\Delta_{n}$ are eliminated in the usual way. If the predicate $Q(n)$ is chosen to be not recursive, we have constructed a system $\Delta^{*}$ of algebraic differential equations involving (among others) the function variables $x_{1}, \cdots, x_{k}, g$, and a polynomial $P\left(n, x_{1}, \cdots, x_{k}, g\right)$ with integer coefficients such that there is no algorithm to determine, given $n$, whether $\Delta^{*}$ has a solution satisfying the boundary condition $P\left(n, x_{1}(1), \cdots, x_{k}(1), g(1)\right)=0$.

III. The uniqueness problem. If the functions are suitably renamed, the system $\Delta_{n}^{\prime}$ of $\S I I$ can be assumed to consist of the equations $D_{i}\left(n, g_{1}, \cdots, g_{m}\right)=0, i=1, \cdots, l$. This system has a solution iff the equation $\sum_{i=1}^{l} D_{i}^{2}=0$ has a solution. Let $\sum_{i=1}^{l} D_{i}^{2}$ $=D\left(n, g_{1}, \cdots, g_{m}\right)$. By inspection of the systems $\Delta_{n}^{\prime}$, if $\left(g_{1}, \cdots, g_{m}\right)$ is a solution, not all the $g_{i}$ are identically 0 .

For a suitable choice of $\Delta_{n}^{\prime}$ there is no algorithm that determines, given $n$, whether the equation $D\left(n, g_{1}, \cdots, g_{m}\right)=0$ has a solution, and hence no algorithm for whether this equation has no solution. But $D\left(n, g_{1}, \cdots, g_{m}\right)=0$ has no solution iff $\left(\sum_{i=1}^{m} g_{i}^{2}\right) D\left(n, g_{1}, \cdots, g_{m}\right)$ $=0$ has a unique solution. Hence we have proved

THEOREM III. There is no uniform procedure for deciding whether a system of algebraic differential equations with integer coefficients has a unique solution in real-valued functions defined on $[0,1]$.

IV. Partial differential equations. In $\S \S I$ and III, our systems have involved several function symbols. Results analogous to 
Theorems II and III can be proved for partial differential equations in only one function symbol. The procedure is very simple. Assume that the equations of the systems $\Delta_{n}^{\prime}$ of $\S I I$ involve the $m$ function symbols $g_{1}, \cdots, g_{m}$. Let $G=G\left(\alpha, \beta_{1}, \cdots, \beta_{m}\right)$. If $G$ satisfies the system of partial differential equations

$$
\partial^{2} G / \partial \beta_{i} \partial \beta_{j}=0, \quad 1 \leqq i \leqq m, 1 \leqq j \leqq m,
$$

then $\partial G / \partial \beta_{i}$ is a function of $\alpha$ alone. If we set $g_{i}=\partial G / \partial \beta_{i}$, and in $\Delta_{n}^{\prime}$ replace differentiation with respect to $\alpha$ by partial differentiation with respect to $\alpha$, then the modified system $\Delta_{n}^{\prime}$ together with (E) has a solution $G$ iff the original system $\Delta_{n}^{\prime}$ has a solution. Recall that the system $D_{i}=0 \quad(1 \leqq i \leqq l)$ has a solution iff the equation $\sum_{i=1}^{l} D_{i}^{2}=0$ has a solution. Then

THEOREM IV. There is no uniform procedure for deciding whether an algebraic partial differential equation with integer coefficients has a solution (or a unique solution).

Other decision problems in analysis can be settled using the techniques of this paper. As an example, we note that it is easy to find functional equations for which $\exp (x)$ and $\sin (x)$ respectively are the only smooth solutions. From this it is relatively easy to show that there is no uniform procedure for deciding whether a system of algebraic functional equations with integer coefficients has continuous solutions.

\section{REFERENCES}

1. M. Davis, Extensions and corollaries of recent work on Hilbert's tenth problem, Illinois J. Math. 7 (1963), 251-256.

2. A. Tarski, $A$ decision method for elementary algebra and geometry, The RAND Corporation, 1948.

INDIANA UNIVERSITY 